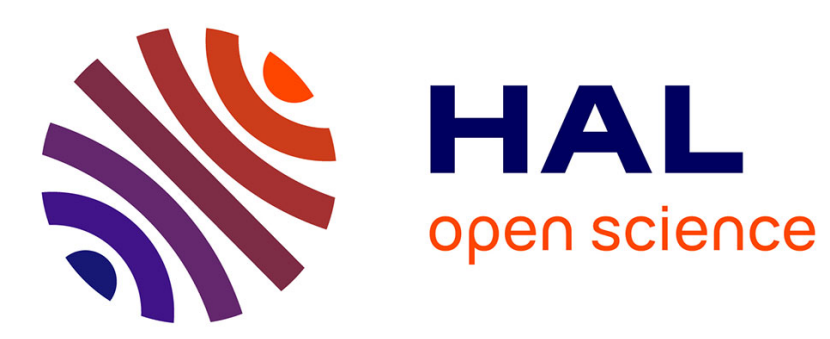

\title{
Les villes à une heure de Paris : des résultats étonnants Gérard-François Dumont
}

\section{To cite this version:}

Gérard-François Dumont. Les villes à une heure de Paris: des résultats étonnants. Population et avenir, 2013, 714, pp.17-19. 10.3917/popav.714.0017 . halshs-00865093

\section{HAL Id: halshs-00865093 https://shs.hal.science/halshs-00865093}

Submitted on 16 Oct 2019

HAL is a multi-disciplinary open access archive for the deposit and dissemination of scientific research documents, whether they are published or not. The documents may come from teaching and research institutions in France or abroad, or from public or private research centers.
L'archive ouverte pluridisciplinaire HAL, est destinée au dépôt et à la diffusion de documents scientifiques de niveau recherche, publiés ou non, émanant des établissements d'enseignement et de recherche français ou étrangers, des laboratoires publics ou privés. 


\section{Les villes à une heure de Paris : des résultats étonnants}

Paris, par son importance démographique, ses fonctions de capitale, ses métiers liés à l'art de vivre et ses points forts touristiques, demeure une ville dont l'attractivité est mondiale. Par effet de proximité, les villes à une heure de Paris en bénéficient-elles ? Pour répondre à cette question, considérons les quatre métropoles ${ }^{1}$ de régions limitrophes de l'Île-de-France ${ }^{2}$ que le réseau ferroviaire met à une heure de Paris $^{3}$ : Amiens, Orléans, Reims et Rouen.

xaminons d'abord les caractéristiques de chacune de ces métropoles en essayant de les classer selon la hiérarchie de leurs avantages objectifs.

\section{Rouen : la plus peuplée et la seule doublement portuaire}

Rouen semble dans la position la plus avantageuse pour plusieurs raisons. D'abord, son unité urbaine compte une population qui est grosso modo le double de celle de chacune des trois autres métropoles régionales considérées. Cela signifie qu'elle présente une polarisation de plus grande importance, pouvant offrir une diversité de population active et un marché de consommateurs beaucoup plus large. Rouen peut donc faire davantage bénéficier les entreprises de ce qu'on appelle les économies d'agglomération, ces économies dont profitent une agglomération et ses acteurs économiques en raison du regroupement, en une même localisation, de plusieurs entreprises appartenant à la même activité ou d'activités différentes sur un même territoire ${ }^{4}$.

Un autre avantage spécifique de Rouen par rapport aux trois autres métropoles tient à son port de fond d'estuaire situé à $110 \mathrm{~km}$ de la mer. Ce port, qui se voit souvent associé à l'image d'un port fluvial, dispose en réalité d'une localisation charnière. À cheval sur la Basse Seine maritime (tronçon entre Rouen et la mer) et la Basse Seine fluviale (tronçon Paris-Rouen), il est un port maritime

\footnotetext{
1. Sur l'ensemble des villes en France, voir : Dumont, Gérard-François (direction), La France en villes, Paris, Sedes, 2010.

2. Lîle-de-France compte une cinquième région limitrophe, la Bourgogne, dont la métropole, Dijon, se trouve à au moins $1 \mathrm{~h} 31$ de Paris par la voie ferrée et à $315 \mathrm{~km}$ par la route, soit une durée de conduite estimée à 3 heures.

3. Une « association des villes à une heure de Paris » avait été créée entre certaines villes concernées en 1994. Reims y avait alors adhéré, avant la réalisation du TGV-Est, alors qu'elle était à $1 \mathrm{~h} 29$ de Paris. Cette association a ensuite changé de nom pour s'appeler "Villes du grand Bassin parisien », considérant que sa première dénomination présupposait que les villes adhérentes se voyaient vouées à devenir de grandes banlieues de Paris ; la nouvelle appellation voulait ainsi renvoyer à une conception non plus concentrique mais multipolaire.

4. Les économies d'agglomération comprennent aussi des économies internes d'échelle propres à une ou plusieurs entreprises.
}

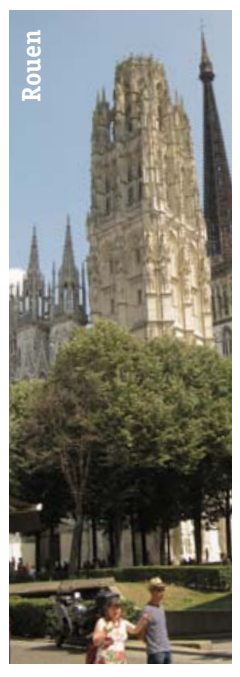

tout autant qu'un port fluvial 5 . En effet, le port de Rouen a la capacité d'accueillir des navires de haute mer dont le tonnage peut atteindre jusquà 18000 tonnes, comme les vraquiers de taille Handysize. Vers l'est, le tronçon fluvial peut atteindre jusquà 3000 tonnes. En outre, le fait que Rouen puisse accueillir des porte-conteneurs de taille petite, voire moyenne, confirme sa nature fluvio-maritime ${ }^{6}$. Dans le contexte de la globalisation et de linternationalisation marquées par l'essor des échanges maritimes, Rouen dispose donc d'un avantage comparatif comme cinquième port français et premier port français pour le transport des céréales.

Une autre caractéristique propre à Rouen, par rapport aux trois autres villes étudiées, est l'existence d'une urbanisation assez continue entre Rouen et Paris, avec un semis de petites agglomérations s'alignant le long de la Seine, sans « trous » importants de peuplement, comme en Beauce, entre Orléans et Paris.

$\begin{array}{cl}\text { par Gérard- } & \text { Rouen bénéficie également d'un important patrimoine } \\ \text { François } & \text { pouvant attirer du tourisme et sa notoriété internationale } \\ \text { Dumont } & \text { se trouve réaffirmée par exemple à chaque fois qu'une nou- } \\ & \text { velle œuvre cinématographique porte sur Jeanne d'Arc. }\end{array}$

1. La distance À Paris de QuATre métropoles DE RÉGIONS LIMITROPHES DE L'ÎLE-DE-FranCE

\begin{tabular}{|l|c|c|c|c|}
\hline $\begin{array}{c}\text { Distance de } \\
\text { Paris à vol } \\
\text { d'oiseau }(\mathrm{km})\end{array}$ & $\begin{array}{c}\text { Distance de } \\
\text { Paris par la } \\
\text { route }(\mathrm{km})\end{array}$ & $\begin{array}{c}\text { Durée de } \\
\text { conduite } \\
\text { estimée par } \\
\text { la route }\end{array}$ & $\begin{array}{c}\text { Durée la plus faible } \\
\text { du trajet ferroviaire } \\
\text { entre Paris et la } \\
\text { gare centre }\end{array}$ \\
\hline Amiens & 116 & 146 & $1 \mathrm{~h} 40$ & $1 \mathrm{~h} 06$ \\
\hline Orléans* & 111 & 133 & $1 \mathrm{~h} 30$ & $1 \mathrm{~h} 05$ \\
\hline Reims & 130 & 144 & $1 \mathrm{~h} 25$ & $0 \mathrm{~h} 46$ \\
\hline * Rph3pour la gare des Aubfais. & 136 & $1 \mathrm{~h} 34$ & $1 \mathrm{~h} 13$ \\
\hline
\end{tabular}

\section{Reims : une notoriété exceptionnelle dans un excellent réseau de transport}


liée à son patrimoine et à son histoire, due en partie à Jeanne d'Arc. Mais son avantage dans ce domaine est supérieur par le renouvellement contemporain de sa notoriété. Dernier exemple : le 8 juillet 2012, le Président de la république française François Hollande et la chancelière de l'Allemagne Angela Merkel ont célébré dans la cathédrale de Reims le $50^{\mathrm{e}}$ anniversaire de la réconciliation franco-allemande, scellée le 8 juillet 1962 par le général de Gaulle et le chancelier allemand Konrad Adenauer.

$\mathrm{Au}$ plan des emplois, Reims pourrait être considérée comme moins pourvue en tertiaire non marchand

5. Verdol, Maïté, «Le "Grand Paris" et ses ouvertures portuaires : Le Havre et Rouen », Population \& Avenir, $n^{\circ} 701$, janvier-février 2011.

6. Le statut de port maritime du port de Rouen a été réaffirmé dans le cadre de la réforme portuaire (loi du 4 juillet 2008) depuis laquelle le port de Rouen possède le statut de Grand Port maritime. 
qu'Amiens, Orléans ou Rouen puisque la capitale de la région Champagne-Ardenne n'est pas Reims, mais Châlons-en-Champagne. Toutefois, d'importantes instances régionales sont installées à Reims, comme le rectorat de l'Académie ou l'Insee. Et Reims demeure la capitale mondiale du champagne, avec des marques très réputées comme Pommery ou Lanson.

Enfin, Reims est la seule des quatre villes étudiées à bénéficier directement d'une liaison TGV. Elle se trouve de ce fait beaucoup plus proche que les autres du premier aéroport français. En effet, depuis 2007, le TGV-Est met Reims à seulement 46 minutes de Paris (gare de l'Est) et à 30 minutes de l'aéroport de Roissy-Charles de Gaulle. Reims compte donc la plus courte distance ferroviaire à Paris des quatre villes étudiées. Elle dispose désormais de deux gares, sa gare de centre-ville et la gare appelée " Champagne-Ardenne TGV » située sur la commune de Bezannes, à $5 \mathrm{~km}$ au sud du centre de Reims. Ce TGV place Reims non seulement dans une position enviable par rapport à Paris, mais aussi au plan européen car ce qu'on appelle en France le TGV-Est est en réalité une ligne à grande vitesse européenne qui a considérablement réduit la durée du transport ferroviaire vers de nombreuses villes européennes, comme Luxembourg, Sarrebruck, Mannheim, Francfort, Karlsruhe, Stuttgart, Munich, Bâle ou Zurich.

Reims se situe donc à portée de la dorsale européenne, ce vaste ensemble allant de Londres à Milan, où l'Europe dispose de sa plus forte densité d'hommes et d'activités. Pour ces raisons, Reims pourrait notamment se considérer comme un lieu d'implantation privilégié des entreprises voulant avoir accès au marché parisien tout en bénéficiant de coûts moindres de fonctionnement ${ }^{7}$. Au plan autoroutier, Reims se trouve à un carrefour entre l'A4 (Paris-Strasbourg) sur l'axe est-ouest et l'A26 (CalaisDijon-Méditerranée) sur l'axe nord-sud. Reims dispose également d'un port, créé en 1948, situé sur le canal de l'Aisne à la Marne, à la lisière du centre-ville et de la desserte autoroutière Est-Ouest. Reims est ainsi connectée au réseau fluvial européen à travers $20000 \mathrm{~km}$ de voies navigables. Toutefois, cela se traduit aujourd'hui par des tonnages transportés relativement faibles.

Reims dispose d'une autre spécificité : l'importance démographique relative de la commune-centre au sein de l'agglomération, ce qui peut en faciliter la gouvernance.

7. Ne subissant donc pas ce qu'on appelle les « déséconomies d'échelle » que peut subir toute entreprise implantée dans une grande agglomération comme celle de Paris.

2. LES CARACTÉRISTIQUES GÉODÉMOGRAPHIQUES

DE QUATRE MÉTROPOLES DE RÉGIONS LIMITROPHES DE L'ILE-DE-FraNCE

\begin{tabular}{|c|c|c|c|c|c|c|c|c|}
\hline & \multicolumn{3}{|c|}{ Commune-centre } & \multicolumn{3}{|c|}{ Unité urbaine } & \multirow{2}{*}{$\begin{array}{l}\text { Poids démo- } \\
\text { graphique } \\
\text { relatif de la } \\
\text { commune- } \\
\text { centre }\end{array}$} & \multirow{2}{*}{$\begin{array}{l}\text { Superficie } \\
\text { relative } \\
\text { de la } \\
\text { commune- } \\
\text { centre }\end{array}$} \\
\hline & $\begin{array}{l}\text { Popula- } \\
\text { tion }\end{array}$ & $\begin{array}{l}\text { Superficie } \\
\left(\mathrm{km}^{2}\right)\end{array}$ & $\begin{array}{c}\text { Densité } \\
\left(\text { hab./ } \mathrm{km}^{2}\right)\end{array}$ & $\begin{array}{l}\text { Popula- } \\
\text { tion }\end{array}$ & $\begin{array}{l}\text { Superficie } \\
\left(\mathrm{km}^{2}\right)\end{array}$ & $\begin{array}{c}\text { Densité } \\
(\text { hab./km²) }\end{array}$ & & \\
\hline Amiens & 133448 & 49 & 2698 & 162718 & 137 & 1185 & $82 \%$ & $36 \%$ \\
\hline Orléans & 114167 & 27 & 4154 & 269724 & 290 & 931 & $42 \%$ & $9 \%$ \\
\hline ms & 992 & 47 & 3837 & 208639 & 94 & 2212 & $86 \%$ & $50 \%$ \\
\hline ouen & 110933 & 21 & 5188 & 463748 & 453 & 1023 & $24 \%$ & $5 \%$ \\
\hline
\end{tabular}

(๑) Gérard-François Dumont - Chiffres Insee RP 2010 (délimitation RP 2010 des unités urbaines).
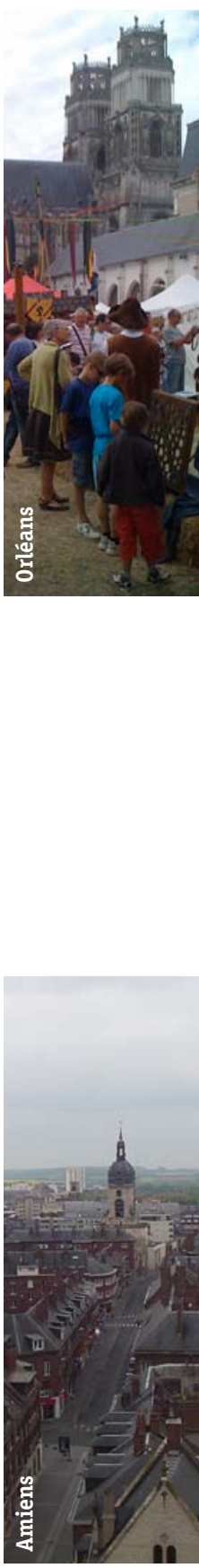

\section{Orléans : une localisation historique aux avantages contemporains limités}

Orléans, ville également marquée par l'histoire, dont notamment et à nouveau, celle de Jeanne d'Arc, compte comme Reims deux gares, mais aucune ne se trouve sur le réseau TGV ${ }^{8}$. Orléans n'a donc pas de relations TGV ni avec Paris, ni avec le reste de la France, ni d'aéroport. Mais la ville offre une patte d'oie autoroutière avec l'A10, qui relie Paris à Tours, Poitiers, puis Bordeaux, et l'A71 qui débute à Orléans pour se diriger vers ClermontFerrand ou permettre de rejoindre l'A20 vers Limoges. Par ailleurs, depuis juin 2009, à $20 \mathrm{~km}$ au nord d'Orléans, l'A10 s'ouvre à une autre voie autoroutière, l'A199 ${ }^{9}$, qui se dirige vers l'Est pour rejoindre notamment, à environ 100 km de là, l'autoroute A6 Paris-Lyon.

La position d'Orléans sur le coude le plus septentrional de la Loire, qui explique l'implantation originelle de cette ville, demeure un avantage limité puisque ce fleuve est très peu navigable, notamment en raison de son débit fort irrégulier. Il existe certes, rive nord du fleuve, partant à proximité du centre ville, le canal d'Orléans, qui se dirige vers l'Est pour rejoindre Montargis, mais ce canal n'est plus en activité dans sa totalité. Sa réhabilitation par le département du Loiret se poursuit pour favoriser le tourisme fluvial

Autre caractéristique : Orléans ne peut être considérée comme la tête de pont de la région Centre dont elle est la capitale politique. En effet, la ville, d'ailleurs bordée par deux vastes espaces largement non constructibles, les forêts d'Orléans au nord-est et de Sologne au sud, demeure moins peuplée que Tours. En outre, le Nord-Est de la région, l'Eure-et-Loir ${ }^{10}$, est plutôt tourné vers Paris et la densité des autres territoires de la région Centre est généralement faible.

\section{Amiens : doublement excentrée}

Dans sa région, Amiens présente certaines caractéristiques faisant penser à Orléans. Il s'agit, outre l'importance de son patrimoine historique, de sa position assez excentrée par rapport à la région Picardie dont elle est capitale. En effet, le Sud de la Picardie, dont les traits géo-historiques correspondent à l'Île-de-France, est largement tourné économiquement vers Paris. De son côté, le Nord du département de l'Aisne, avec Saint-Quentin, vit plutôt en symbiose avec la région Nord-Pas-de-Calais, conformément au découpage administratif régional que proposait le grand géographe Paul Vidal de La Blache en $1910^{11}$. Et l'Est de la Picardie se sent proche de Reims. Au plan ferroviaire, Amiens, comme Rouen et Orléans, ne dispose pas de gare TGV. Certes, le TGV Nord ParisLille comprend depuis 1993 une gare TGV Haute-Picardie, bâtie en rase campagne et surnommée à juste titre la gare des betteraves, mais cette gare, qui se trouve à $39 \mathrm{~km}$ d'Amiens, n'est nullement reliée à cette ville par une voie ferroviaire. Amiens connaît une autre spécificité peu favorable : sa région est celle qui, chaque année, compte

8. Le TGV Paris-Tours passe par Vendôme, contrairement à l'autoroute A10 qui passe par Orléans.

9. Dont l'objet à terme serait un grand contournement de Paris.

10. «Territoire au banc d'essai : l'Eure-et-Loir », Population \& Avenir, hors série, $n^{\circ}$ 659bis, septembre-octobre 2002.

11. "Régions françaises », La revue de Paris, 15 décembre 1910 (numéro de novembre-décembre). 
les résultats scolaires les moins favorables. Notons aussi qu'Amiens est la moins peuplée des quatre villes étudiées et, donc, celle qui peut normalement offrir le moins d'économies d'agglomération. Enfin, sa notoriété est moindre.

Ainsi, ces quatre métropoles se trouvent en situation semblable par le fait d'être à une heure de Paris, ce qui explique une partie parfois importante de leurs activités, mais aussi une dépendance aux stratégies de sièges sociaux parisiens, et des migrations alternantes. Toutefois, la synthèse de leurs caractéristiques met en évidence de nombreuses inégalités territoriales qui devraient conduire à les classer en terme d'attractivité dans l'ordre suivant : Rouen, Reims, Orléans et Amiens.

\section{Des résultats inattendus}

Or, si l'on mesure l'attractivité par l'évolution démographique depuis le recensement de 1975 à l'échelle des unités urbaines ${ }^{12}$ de ces quatre villes, donc depuis le grand tournant économique qui a vu la fin d'une ère essentiellement industrielle, le résultat ne correspond nullement à cet ordre. Nos quatre villes se répartissent en deux types. D'une part, une ville, Orléans, a connu, du recensement de 1975 à celui de 2010, une importante croissance démographique, nettement supérieure à la moyenne nationale et constamment positive lors des différentes périodes intercensitaires. D'autre part, les accroissements démographiques des trois autres villes sont demeurés nettement inférieurs à la moyenne nationale, et même parfois négatifs lors de certaines périodes intercensitaires.

Comme le tiers de siècle considéré (1975-2010) a été marqué par une augmentation de l'espérance de vie, la légère augmentation de la population des unités urbaines de Rouen, Reims et Amiens s'apparente à une stagnation. Le fait d'être à une heure de Paris, voire moins dans le cas de Reims, n'apparaît pas comme un avantage engendrant automatiquement de l'attractivité. $\mathrm{Si}$, au lieu de considérer l'agglomération des villes correspondant au concept d'unité urbaine de l'Insee, nous examinons la commune-centre, le résultat est semblable : le plus fort accroissement 1975-2010 des quatre communes-centres s'observe pour la commune d'Orléans et le plus faible, négatif, concerne Rouen. Une comparaison à l'échelle des aires urbaines, selon la délimitation 2010 de l'Insee, donne le même résultat d'ensemble : une croissance démographique de $37 \%$ pour l'aire urbaine d'Orléans de 1975 à 2010 et des croissances démographiques comprises entre $11 \%$ et $16 \%$ pour les autres aires urbaines, donc inférieures à la moyenne de la France métropolitaine $(19 \%)$

Les différences d'attractivité des quatre métropoles régionales situées à une heure de Paris ne peuvent s'expliquer par leurs caractéristiques objectives, géohistoriques ou géographiques. Pour trois d'entre elles, le résultat se présente comme si la proximité avec Paris n'engendrait aucun avantage, puisque leur accroissement démographique est moindre que la moyenne nationale. Comment, alors, expliquer les résultats atypiques de l'une d'entre elles, Orléans?

12. Les résultats proposés ici concernent l'unité urbaine des villes, dans leur dernière délimitation 2010, ce qui signifie qu'ils intègrent les effets de la périurbanisation des années 1975 à 2010.

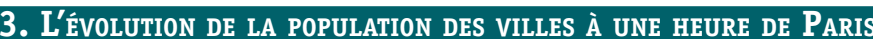

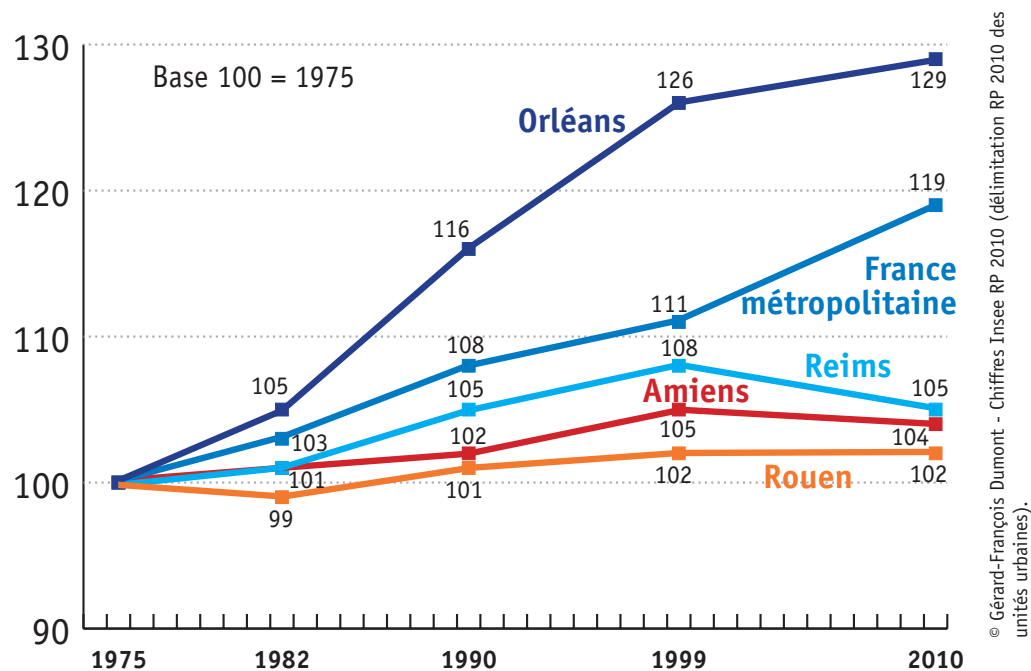

D'abord ${ }^{13}$, alors que les trois autres villes semblent continuer à s'appuyer pour une part importante sur des atouts économiques anciens, avec parfois une tendance à trop s'inscrire dans une économie de rente, il semble qu'Orléans s'est davantage positionnée dans l'industrie de la connaissance, a plus développé des activités de haute technologie. L'investissement dans l'innovation, ce qui suppose également d'autres investissements (culturels, urbanistiques...) pour satisfaire la demande des acteurs économiques qui aspirent à une bonne qualité de la vie, permet une meilleure attractivité relative. En revanche, quand des activi-
Le fait d'être à une heure de Paris n'apparait pas comme un avantage engendrant automatiquement de l'attractivité.

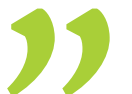
tés économiques plus traditionnelles occupent une place importante, l'attractivité apparaît moindre.

Un autre facteur explicatif de l'attractivité différenciée tient au degré de diversification de l'économie, qui est plus élevé à Orléans, avec quatre secteurs importants : la pharmacie et la parachimie, l'agroalimentaire (stimulé par la proximité de la Beauce), la mécanique et l'électronique. Lactivité économique s'y organise autour d'établissements installés par des firmes multinationales et de nombreuses PMI, sans qu'il y ait d'entreprises dominantes. Orléans est aussi présente dans le secteur tertiaire, avec des services directionnels, comme des fonctions financières de La Poste, dont le centre de chèques postaux de la Source. Le tertiaire supérieur a commencé de se déployer, en particulier les services aux entreprises, avec des écoles d'ingénieurs, des chercheurs en agroalimentaire ou en matériaux composites et des laboratoires décentralisés. Enfin, Orléans dispose d'un pôle logistique en raison de sa situation de carrefour autoroutier à proximité de Paris.

L'exemple des villes situées à une heure de Paris confirme l'absence de corrélation entre l'analyse des forces et des faiblesses objectives des villes et leur attractivité. Ce qui justifie de prendre en considération d'autres éléments essentiels, comme la gouvernance ${ }^{14}$ et son évolution.

13. Par ailleurs, le rôle du quartier d'Orléans-la-Source, fondé dans les années 1960, d'une conception urbanistique proche des villes nouvelles, doit être écarté car il a connu sa forte croissance démographique avant 1975. En revanche, Il faut noter que la fécondité du Loiret est supérieure à celle des départements des trois autres villes : voir " La démographie de la France en 2010 : le double paradoxe ", Population \& Avenir, $\mathrm{n}^{\circ} 702$, mars-avril 2011.

14. Dumont, Gérard-François, Diagnostic et gouvernance des territoires, Paris, Armand Colin, 2012. 